\title{
Growth of boron-doped few-layer graphene by molecular beam epitaxy
}

G. V. Soares, S. Nakhaie, M. Heilmann, H. Riechert, and J. M. J. Lopes

Citation: Appl. Phys. Lett. 112, 163103 (2018); doi: 10.1063/1.5019352

View online: https://doi.org/10.1063/1.5019352

View Table of Contents: http://aip.scitation.org/toc/apl/112/16

Published by the American Institute of Physics

\section{Articles you may be interested in}

Electrostatic properties of graphene edges for electron emission under an external electric field

Applied Physics Letters 112, 163105 (2018); 10.1063/1.5023845

Origin and roles of oxygen impurities in hexagonal boron nitride epilayers

Applied Physics Letters 112, 162103 (2018); 10.1063/1.5026291

Van der Waals epitaxy of two-dimensional single-layer h-BN on graphite by molecular beam epitaxy: Electronic properties and band structure

Applied Physics Letters 112, 253102 (2018); 10.1063/1.5029220

Invisible magnetic sensors

Applied Physics Letters 112, 162406 (2018); 10.1063/1.5023565

Scalable and reusable micro-bubble removal method to flatten large-area 2D materials

Applied Physics Letters 112, 163106 (2018); 10.1063/1.5022057

Crystal-phase intergradation in InAs nanostructures grown by van der Waals heteroepitaxy on graphene Applied Physics Letters 112, 142101 (2018); 10.1063/1.5017251

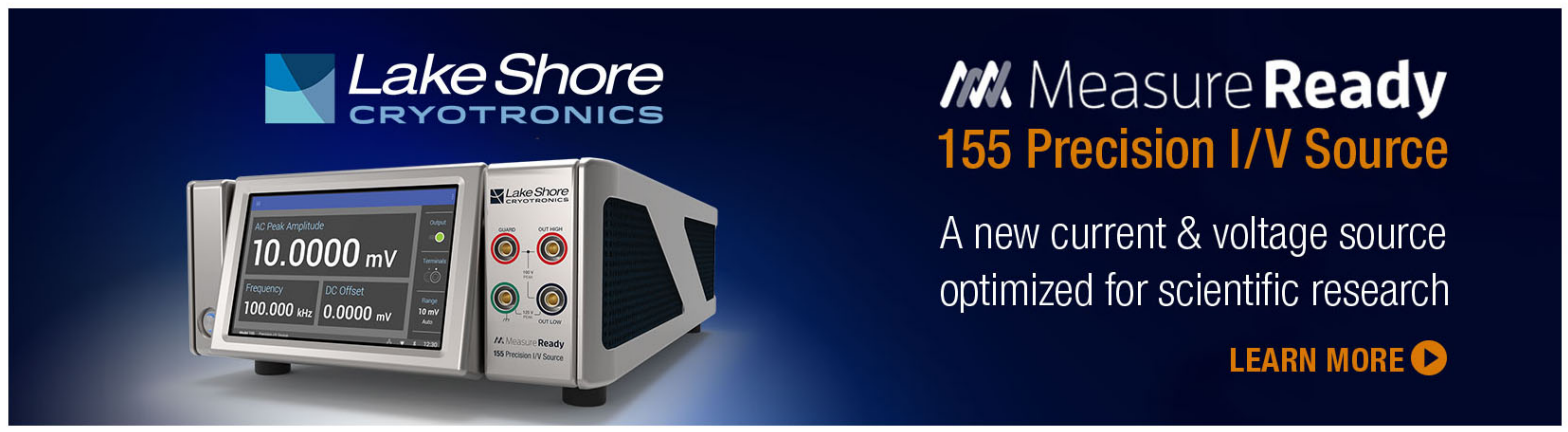




\title{
Growth of boron-doped few-layer graphene by molecular beam epitaxy
}

\author{
G. V. Soares, ${ }^{1, a), b)}$ S. Nakhaie, ${ }^{2}$ M. Heilmann, ${ }^{2}$ H. Riechert, ${ }^{2}$ and J. M. J. Lopes ${ }^{2}$ \\ ${ }^{1}$ Instituto de Física, UFRGS, Porto Alegre, RS 91501-970, Brazil \\ ${ }^{2}$ Paul-Drude-Institut für Festkörperelektronik, Leibniz-Institut im Forschungsverbund Berlin e.V., \\ Hausvogteiplatz 5-7, 10117 Berlin, Germany
}

(Received 13 December 2017; accepted 3 April 2018; published online 17 April 2018)

\begin{abstract}
We investigated the growth of boron-doped few-layer graphene on $\alpha-\mathrm{Al}_{2} \mathrm{O}_{3}(0001)$ substrates by molecular beam epitaxy using two different growth approaches: one where boron was provided during the entire graphene synthesis and the second where boron was provided only during the second half of the graphene growth run. Electrical measurements show a higher p-type carrier concentration for samples fabricated utilizing the second approach, with a remarkable modulation in the carrier concentration of almost two orders of magnitude in comparison to the pristine graphene film. The results concerning the influence of the boron flux at different growth stages of graphene on the electrical and physicochemical properties of the films are presented. Published by AIP Publishing.

https://doi.org/10.1063/1.5019352
\end{abstract}

Graphene has attracted much attention in the last few years due to its outstanding properties such as high mobility of charge carriers $\left(\mu>100000 \mathrm{~cm}^{2} / \mathrm{Vs}\right.$ even at room temperature), ${ }^{1}$ high mechanical strength, ${ }^{2}$ high thermal conductivity, ${ }^{3}$ large specific surface area, and optical transparency. ${ }^{4}$ These properties make this material very attractive for a wide range of applications including high-frequency transistors, touch and flexible screens, ultra-sensitive sensors for gas, strain and chemicals, and electrochemical energy applications. ${ }^{5}$ However, limitations and challenges still remain in order to employ graphene with its full potential in these applications. For instance, one of the main issues for electronic applications is the fact that graphene is a zero bandgap material. Substitutional doping is among the most efficient and controlled methods to tailor the properties of graphene. Previous works have shown that the introduction of nitrogen dopants can open a bandgap in graphene $e^{6,7}$ and also increase its carrier concentration. ${ }^{8}$ Nitrogen and boron can induce $n$ type and p-type doping in graphene, respectively, but unintentional p-type doping can occur in pristine graphene due to species absorbed from the atmosphere such as oxygen and water vapor. ${ }^{9}$ Since uncontrolled p-type doping is easily achieved in graphene, a higher research effort was devoted to nitrogen-doping in order to produce n-type graphene. However, it remains mandatory to modulate graphene electronic properties by controlled doping of the p-type and/or the n-type and at the same time to avoid the presence of undesirable contaminants. In this way, the investigation of boron-doped graphene has gained increasing attention. Boron-doped graphene has been shown to be of high utility in several applications including hydrogen storage $^{10}$ and oxygen reduction in fuel cells, ${ }^{11}$ in supercapacitor electrodes due to the high electrical conductivity and large surface area, ${ }^{12}$ and in ultrasensitive gas sensors. ${ }^{13}$ Different methods have been employed to synthesize boron-doped graphene, including chemical vapor deposition (CVD), ${ }^{13}$ thermal

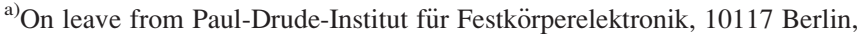
Germany.

b) Author to whom correspondence should be addressed: gabriel.soares@ ufrgs.br
}

annealing, ${ }^{14}$ and micromechanical exfoliation of borondoped graphite. ${ }^{15}$ Despite the significant progress that has been achieved, the controlled growth of B-doped graphene on a wide variety of substrates, such as dielectrics and/or semiconductors, remains a challenge.

A growth method that offers potential to achieve this task is molecular beam epitaxy (MBE), which is a wellestablished technique for the production of high-quality single crystal semiconductors and doped semiconductor layers. ${ }^{16}$ MBE has also been used to grow graphene layers on several substrates with different degrees of success ${ }^{17-20}$ but has not been explored for graphene doping. The possibility of growing graphene directly on a dielectric substrate and of achieving precise control over thickness and doping levels makes MBE a promising technique for the synthesis of dopedgraphene. In the present work, we report on the MBE growth of boron-doped few-layer graphene on $\alpha-\mathrm{Al}_{2} \mathrm{O}_{3}$ (0001) substrates. Boron doping of few-layer graphene was investigated following two different growth approaches: one where boron was added during the entire graphene synthesis and the second where boron was added only during the second half of the graphene growth run. Although the method (1) resulted in a higher concentration of boron atoms in the graphene films, the latter achieved a higher p-type carrier concentration. The results concerning the influence of the boron flux at different stages of few-layer graphene growth on the electrical and physicochemical properties of the graphene films will be presented.

Starting samples were $1 \mathrm{~cm} \times 1 \mathrm{~cm}$ large $\alpha-\mathrm{Al}_{2} \mathrm{O}_{3}$ (0001). The high thermal stability of $\mathrm{Al}_{2} \mathrm{O}_{3}$ allows us to use high sample temperatures during MBE growth. The substrates were back-coated with $1 \mu \mathrm{m}$ thick Ti (to allow for non-contact heating by radiation), cleaned using standard solvents, and loaded in the preparation chamber of the MBE system for outgassing at $300^{\circ} \mathrm{C}$ for $1 \mathrm{~h}$. Finally, the substrates were transferred to the growth chamber, which is equipped with an e-beam evaporator filled with a pyrolytic graphite target (for $\mathrm{C}$ evaporation) and a high-temperature effusion cell for evaporation of elemental B. The samples were heated up to $950^{\circ} \mathrm{C}$ (the temperature was determined 
using a pyrometer at a wavelength of $940 \mathrm{~nm}$ ), and then, a power of $750 \mathrm{~W}$ was applied to the e-beam. Under these conditions (at a pressure of $5 \times 10^{-8} \mathrm{mbar}$ ), a $\sim 3$ monolayer (ML) thick graphene film is grown after $10 \mathrm{~min}$, as determined by atomic force microscopy profile measurements taken at the edges of the film. Boron was introduced into the films by two different approaches: a one-step doping approach (here after called 1), where the boron and carbon source shutters were opened simultaneously and the growth/ doping process was kept for $10 \mathrm{~min}$; and a two-step doping approach (here after called 2), where the $\mathrm{Al}_{2} \mathrm{O}_{3}$ surface was exposed only to the $\mathrm{C}$ beam for an interval of $5 \mathrm{~min}$, followed by another $5 \mathrm{~min}$ interval in which $\mathrm{B}$ is added to the process. This yields a total growth time of $10 \mathrm{~min}$, as the one used in approach 1. The two approaches were compared taking into account the influence of the employed boron flux (varied from $\leq 6.8 \times 10^{11}$ to $1.5 \times 10^{13}$ atoms $/ \mathrm{cm}^{2} \mathrm{~s}$ ) in the electrical and physicochemical properties of synthesized layers. In order to modulate the boron flux, the temperature of the effusion cell utilized for B evaporation was varied from $1550{ }^{\circ} \mathrm{C}$ to $1950^{\circ} \mathrm{C}$. Please note that the lowest B flux that could be detected by quadrupole mass spectrometry in an UHV test chamber operating at a base pressure of $\sim 10^{-9}$ mbar was $6.8 \times 10^{11}$ atoms $/ \mathrm{cm}^{2} \mathrm{~s}$, for a corresponding cell temperature of $1750^{\circ} \mathrm{C}$.

Raman spectroscopy analyses were carried out using a $473 \mathrm{~nm}$ wavelength laser. The spectra were obtained with a spatial resolution of $1 \mu \mathrm{m}$. Several measurements performed at different locations on the sample surface allowed for checking the layer homogeneity. X-ray photoelectron spectroscopy (XPS) measurements were performed using an Al $\mathrm{K} \alpha \mathrm{X}$-ray source. The samples were outgassed at $250^{\circ} \mathrm{C}$ for $30 \mathrm{~min}$ prior to the XPS measurements. The carrier density and mobility were obtained by magnetotransport measurements in a van der Pauw geometry at room temperature and at a magnetic field of $0.6 \mathrm{~T}$.

Figure 1 shows the XPS measurements of the B 1s energy region. XPS was employed to confirm the presence of $\mathrm{B}$ and to identify the chemical environment of the B atoms. The spectrum of the film grown for $10 \mathrm{~min}$ (without boron), hereafter called non-doped, is presented with full black circles, where no visible signal of boron can be detected (within the sensitivity of 1 at. \%). The data for approaches 1 (triangles) and 2 (squares) are also plotted, where in both cases the highest boron cell flux and temperature $\left(1.5 \times 10^{13}\right.$ atoms $/ \mathrm{cm}^{2} \cdot \mathrm{s}$ and $1950^{\circ} \mathrm{C}$, respectively) were employed. By analyzing the survey spectra (data not shown) of approaches 1 and 2, we determined the boron atomic concentration in the graphene film as 10 at. $\%$ and 7.6 at. \%, respectively. Three different bonding configurations are observed: boron bonded to carbon (at $186.5 \mathrm{eV}$, red line), ${ }^{21}$ to boron (at $187.6 \mathrm{eV}$, blue line), ${ }^{21}$ and to oxygen $(189.2 \mathrm{eV}$, pink line). ${ }^{22}$ Boron bonded to carbon one can be attributed to substitutional boron within the graphene lattice and/or bonded to graphene domain boundaries. The second can be due to interstitial boron in the graphene film or boron located at the surface. The third contribution is probably related to a partial oxidation of this boron and/or of edge-bonded boron ${ }^{13}$ when the sample is exposed to the atmosphere (between MBE growth and the XPS measurement). Interestingly,

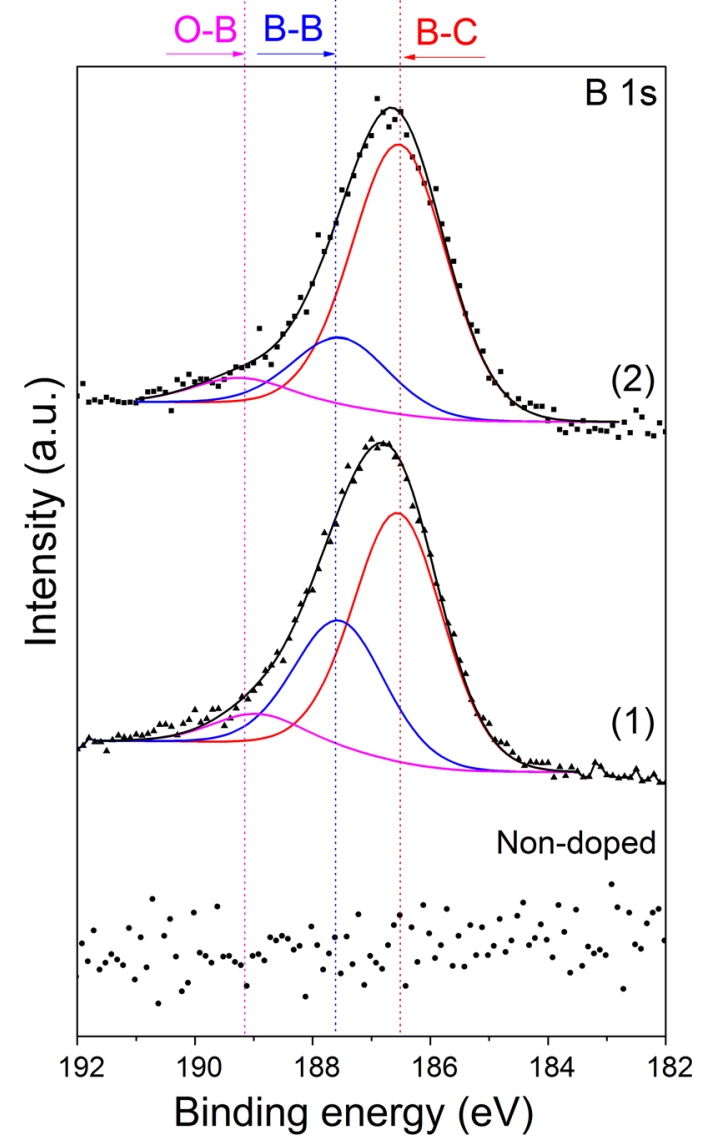

FIG. 1. X-ray photoelectron spectroscopy of the B 1s energy region for the following samples: non-doped (circles) graphene and B-doped graphene obtained by approaches 1 (triangles) and 2 (squares). The main energy regions for boron bonded to carbon (red), to boron (blue), and to oxygen (pink) are indicated. A.u. stands for arbitrary units.

although approach 2 uses only half of the B nominal dose used in approach 1 (in practice, XPS shows that approach 1 leads to a $\sim 30 \%$ higher $\mathrm{B}$ total incorporation), the sample prepared using approach 2 shows a larger fraction $(\sim 80 \%)$ of boron bonded to carbon when compared to the one obtained via approach $1(\sim 50 \%)$. This reveals that boron is more efficiently incorporated in the graphene lattice as an impurity when the MBE growth approach 2 is employed. This result is intriguing, and the origin is not clear at this moment and requires further investigation. Nevertheless, we consider as a logical assumption that this behavior is directly related to the growth mechanisms of graphene on sapphire. It is known that the growth process involves two main stages: nucleation of islands followed by their lateral growth and coalescence. In the growth approach 2, boron is provided only after the graphene nucleation already took place and at least a monolayer of graphene covers the surface. For approach 1, boron is provided during the entire growth process. Hence, in terms of graphene nucleation and growth and B incorporation, approach 1 represents a substantially different and likely more complex scenario which can result in a less efficient incorporation of boron impurity atoms in the graphene lattice.

In Figure 2, the Raman spectra collected for samples prepared with different boron fluxes using approaches 1 (left-hand side) and 2 (right-hand side), as well as for the non-doped graphene, are shown. It is possible to observe 


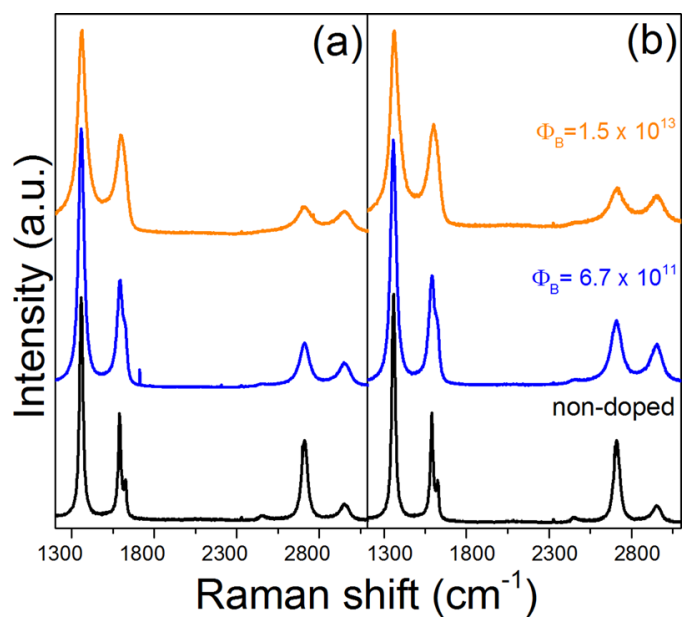

FIG. 2. Raman spectra for the non-doped sample (black) and samples doped using the indicated boron fluxes, for approaches 1 (a) and 2 (b). $\Phi_{\mathrm{B}}$ stands for boron flux in atoms $/ \mathrm{cm}^{2} \cdot \mathrm{s}$ units. A.u. stands for arbitrary units.

several features in the Raman spectra, which confirms the growth of graphene. By analyzing the spectra, we identify the $\mathrm{G}$ peak around $\sim 1580 \mathrm{~cm}^{-1}$ attributed to the presence of $\mathrm{sp}^{2}$ bonded carbon atoms. The D peak localized at around $1350 \mathrm{~cm}^{-1}$ and the $\mathrm{D}^{\prime}$ peak at $\sim 1620 \mathrm{~cm}^{-1}$ are related to a disorder-induced mode due to the existence of point defects, domain boundaries, and dopants. Finally, at $\sim 2700 \mathrm{~cm}^{-1}$, the $2 \mathrm{D}$ peak is observed. The presence of these four peaks is consistent with the growth of nanocrystalline few-layer graphene films on $\mathrm{Al}_{2} \mathrm{O}_{3}(0001)$ by MBE. ${ }^{18,20,23}$ The thickness of the resulting graphene film was estimated using atomic force microscopy (data not shown), yielding a $1 \mathrm{~nm}$ thick film. This is approximately 3 layers of graphene (a graphene single layer has a thickness of $3.35 \AA$ ). However, we do not observe the characteristic asymmetric shape of the 2D peak associated with $\mathrm{AB}$-stacked trilayer graphene ${ }^{24}$ but rather a symmetric shape that might be associated with a turbostratic stacking arrangement between the graphene adjacent layers. ${ }^{18}$ When boron is added during growth using both approaches, we observe systematic changes in the Raman spectra, such as a blue shift in the $G$ and $2 D$ peaks and a reduction of the 2D peak intensity. Figures 3(a) and 3(b) show the $2 \mathrm{D}$ and $\mathrm{G}$ peak positions for doping approaches 1 and 2 as a function of the boron cell temperature and flux, respectively. In both cases, a blue shift is observed, which can be attributed mainly to the boron doping effect in graphene as the p-type. ${ }^{8,25} \mathrm{G}$ peak shifts of up to 8 and $10 \mathrm{~cm}^{-1}$ are observed for the films prepared using approaches 1 and 2 under the highest $\mathrm{B}$ flux, respectively. For the 2D peak, the corresponding values are 6.5 and $7.5 \mathrm{~cm}^{-1}$, respectively.

Please note that boron incorporation should lead to local strain in the graphene lattice which can also promote a shift in the $\mathrm{G}$ and $2 \mathrm{D}$ peaks. ${ }^{26}$ However, in our discussion, we consider the doping effect in the $\mathrm{G}$ and $2 \mathrm{D}$ peak positions to be more pronounced than the mechanical one, which is corroborated by our electrical results presented in Fig. 4.

The intensity of the $G$ and $2 \mathrm{D}$ peaks also changes with doping. Particularly, the 2D peak intensity decreases with the shift of the Fermi level position. This can be observed by considering the ratio between the areas of the $2 \mathrm{D}$ and $\mathrm{G}$ peaks [see Fig. 3(c)]. A decrease in $\mathrm{A}_{2 \mathrm{D}} / \mathrm{A}_{\mathrm{G}}$ takes place as

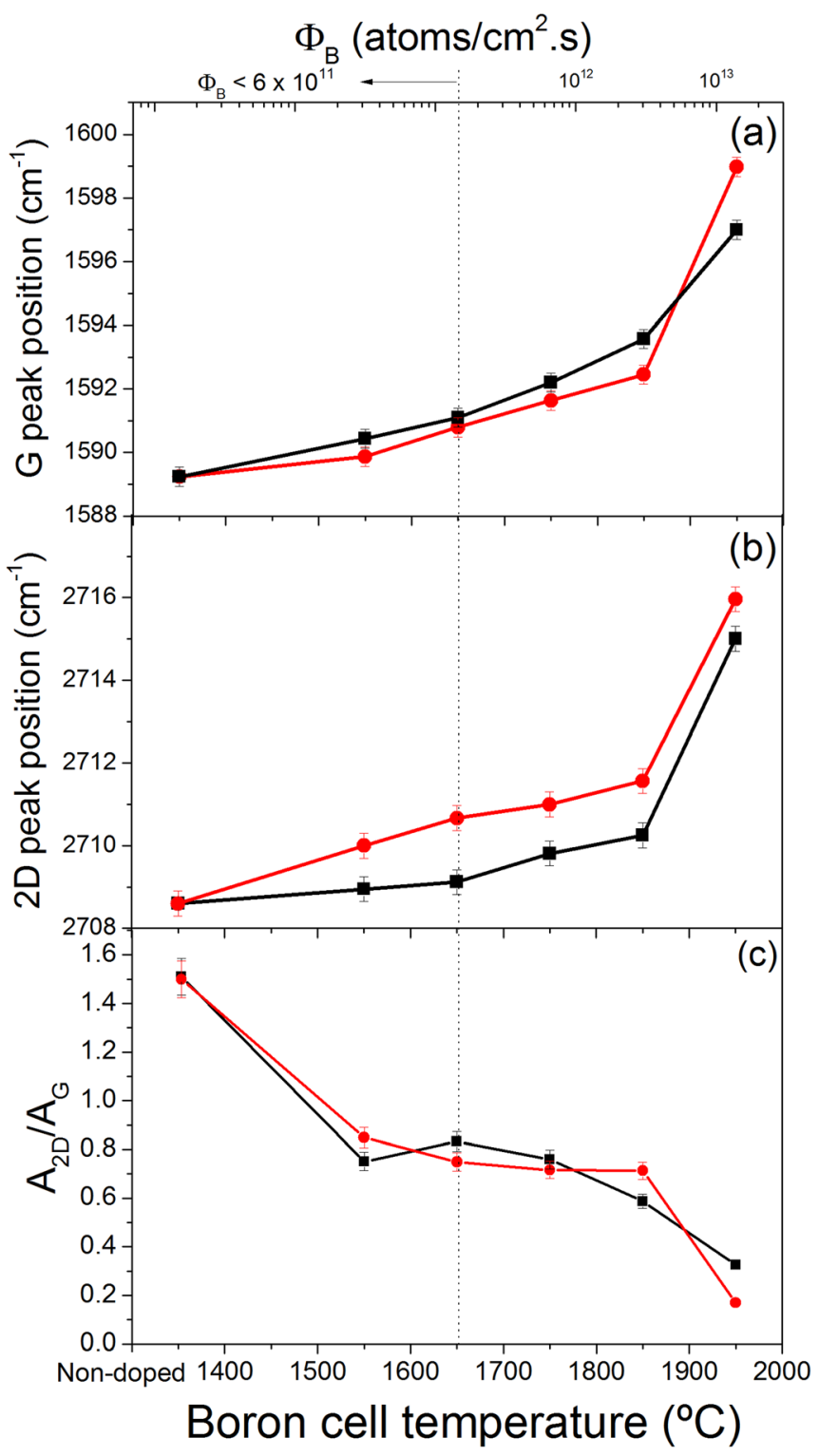

FIG. 3. (a) $2 \mathrm{D}$ and (b) G peak positions and the (c) 2D to G peak area ratio, plotted as a function of the boron cell temperature (bottom X-axis) and boron flux (top X-axis) for doped graphene films synthesized using approaches 1 (black squares) and 2 (red circles). The lines are only to guide the eyes.

the utilized boron flux increases, a behavior that is compatible with an increase in the doping level in the graphene film. Previous work ${ }^{25}$ reported that a $2 \mathrm{D} / \mathrm{G}$ ratio lower than 0.6 , which is obtained for boron fluxes higher than $6.7 \times 10^{11}$ atoms $/ \mathrm{cm}^{2} \cdot \mathrm{s}$, can be related to a carrier concentration $\mathrm{N}_{\mathrm{p}}>4$ $\times 10^{13} \mathrm{~cm}^{2}$. This is consistent with the electrical measurements that will be presented later.

In general, the Raman results of the samples prepared using approaches 1 and 2 seem to indicate, in particular for the films synthesized under the highest B flux, that the 2 steps of the growth approach result into a higher carrier concentration. This is in agreement with the XPS data shown in Fig. 1, where a higher portion of substitutional boron is obtained using approach 2.

Figure 4(a) shows the carrier concentration as a function of boron cell temperature/flux for the samples prepared using approaches 1 (black squares) and 2 (red circles). The non-doped graphene film exhibits a carrier concentration of 


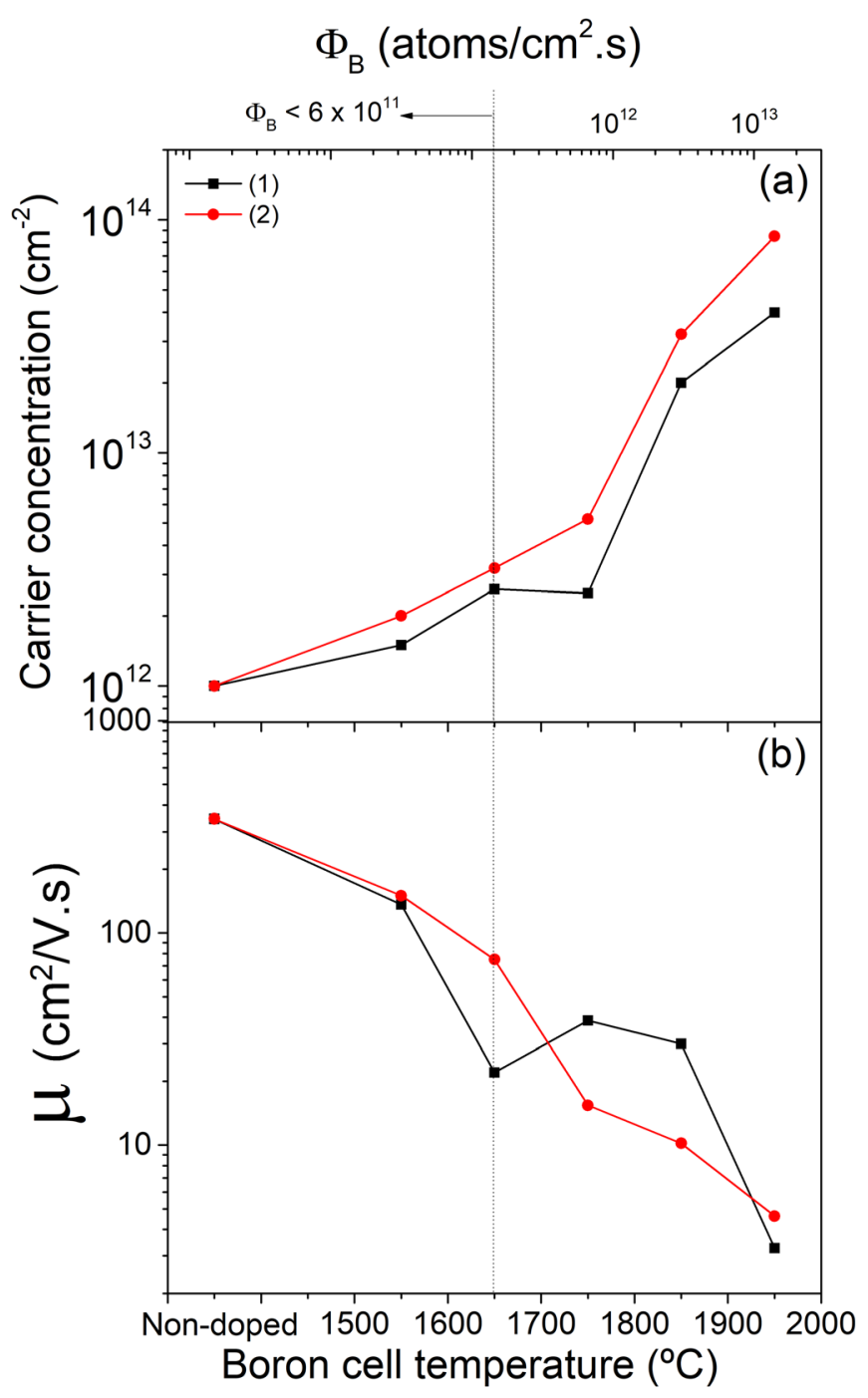

FIG. 4. (a) Charge carrier concentration and (b) charge carrier mobility as a function of the boron cell temperature (bottom X-axis) and boron flux (top $\mathrm{X}$-axis) doped graphene films synthesized using approaches 1 (black squares) and 2 (red circles). The lines are only to guide the eyes.

$\sim 1 \times 10^{12} \mathrm{~cm}^{-2}$, with p-type doping. This doping type is consistent with previous reports for graphene on $\mathrm{Al}_{2} \mathrm{O}_{3}$ prepared either by CVD or MBE. ${ }^{18,20,27}$ Increasing boron flux leads to an increase in the carrier concentration for both approaches, where the carrier concentration is higher for approach 2 for all investigated fluxes. For a flux of $1.5 \times 10^{13}$ atoms $/ \mathrm{cm}^{2} \cdot \mathrm{s}$, a maximum of $8 \times 10^{13} \mathrm{~cm}^{-2}$ and $4 \times 10^{13}$ $\mathrm{cm}^{-2}$ for approaches 2 and 1 , respectively, has been found. The higher carrier concentration obtained for approach 2 is corroborated by the other results, namely, a higher concentration of boron bonded to carbon (as show by XPS) and a higher blue shift of the G and 2D Raman peaks. This suggests that approach 2 is more efficient than approach 1 to achieve growth of B-doped graphene via MBE.

Figure 4(b) shows the charge carrier mobility as a function of the boron cell temperature/flux. The pristine film exhibits a mobility of $320 \mathrm{~cm}^{2} / \mathrm{V} \cdot \mathrm{s}$, which is higher than what was previously reported by our group also for graphene grown on $\mathrm{Al}_{2} \mathrm{O}_{3}(0001)$ using MBE. ${ }^{18,20}$ This is probably due to the lower carrier concentration of the pristine graphene films studied here $\left(\sim 10^{12}\right.$ instead of $\sim 10^{13} \mathrm{~cm}^{-2}$ as reported in $^{18,20}$ ) and/or to the lower density of defects present in the few-layer graphene of this work. The reason behind the improvement in the electrical properties (when compared to previous works) can be the employment of different conditions for the carbon source (e.g., the higher carbon flux utilized in this work). It is expected that the choice of source and the corresponding operation conditions can impact the properties of the resulting MBE-grown graphene. ${ }^{28}$ Finally, for the doped films, a similar trend is observed for samples prepared using growth approaches 1 and 2: a reduction in mobility when boron flux is increased, which is anticipated due to the creation of a higher number of scattering centers in the graphene lattice. However, differently from the charge carrier concentration, no clear distinction between the values obtained for samples prepared by approaches 1 and 2 can be seen. This might be related to the complex scenario involving charge carrier scattering in these samples. Given the fact that, besides the incorporation of B in the films, other structural imperfections such as point defects and grain boundaries will also contribute to a decrease in mobility, even small fluctuations in the formation and interplay between these type of defects during growth and $\mathrm{B}$ incorporation (e.g., filling of vacancies by $\mathrm{B}$ atoms and modification of the grain boundary regions due to $\mathrm{B}$ incorporation) could be one of the reasons behind the variability observed in mobility.

In summary, we investigated the boron doping of fewlayer graphene grown by $\mathrm{MBE}$ on $\mathrm{Al}_{2} \mathrm{O}_{3}(0001)$. Two different doping approaches were employed, while the boron flux was varied in a wide range: one with a simultaneous growth/ doping of graphene and the second with a nucleation step followed by a growth/doping step. The XPS results show the incorporation of boron in the graphene lattice. Raman analysis indicates a systematic increase in the graphene p-type doping level when boron emission is increased. Electrical measurements demonstrate a higher carrier concentration for approach 2 , which indicates a more efficient doping activation when compared to approach 1. Finally, a remarkable modulation in the carrier concentration in graphene could be obtained (almost two orders of magnitude), which is relevant for several applications in electronic devices and electrochemistry.

G.V.S. would like to acknowledge CAPES-Humboldt fellowship number 1675-14-1. The authors would like to thank J.M. Wofford for fruitful discussions and H.-P. Schönherr, M. Höricke, and C. Hermann for their dedicated maintenance of the MBE system. Furthermore, the authors appreciate the critical reading of the manuscript by Stefano Cecchi.

${ }^{1}$ A. K. Geim and K. S. Novoselov, Nat. Mater. 6, 183 (2007).

${ }^{2}$ C. Lee, X. Wei, J. W. Kysar, and J. Hone, Science 321(5887), 385-388 (2008).

${ }^{3}$ A. A. Balandin, S. Ghosh, W. Bao, I. Calizo, D. Teweldebrhan, F. Miao, and C. N. Lau, Nano Lett. 8, 902 (2008).

${ }^{4}$ G. Eda, G. Fanchini, and M. Chhowalla, Nat. Nanotechnol. 3, 270 (2008).

${ }^{5}$ A. C. Ferrari, F. Bonaccorso, V. Falko, K. S. Novoselov, S. Roche, P. Bøggild, S. Borini, F. H. L. Koppens, V. Palermo, N. Pugno et al., Nanoscale 7, 4598 (2015).

${ }^{6}$ D. Usachov, O. Vilkov, A. Grüneis, D. Haberer, A. Fedorov, V. K. Adamchuk, A. B. Preobrajenski, P. Dudin, A. Barinov, M. Oehzel et al., Nano Lett. 11, 5401 (2011).

${ }^{7}$ D. Wei, Y. Liu, Y. Wang, H. Zhang, L. Huang, and G. Yu, Nano Lett. 9, 1752 (2009). 
${ }^{8}$ G. Sarau, M. Heilmann, M. Bashouti, M. Latzel, C. Tessarek, and S. Christiansen, ACS Appl. Mater. Interfaces 9, 10003 (2017).

${ }^{9}$ P. Joshi, H. E. Romero, A. T. Neal, V. K. Toutam, and S. A. Tadigadapa, J. Phys.: Condens. Matter 22, 334214 (2010).

${ }^{10}$ S. Nachimuthu, P. J. Lai, E. G. Leggesse, and J. C. Jiang, Sci. Rep. 5, 16797 (2015).

${ }^{11}$ L. Wang, H. Dong, Z. Guo, L. Zhang, T. Hou, and Y. Li, J. Phys. Chem. C 120, 17427 (2016).

${ }^{12}$ J. Han, L. L. Zhang, S. Lee, J. Oh, K. S. Lee, J. R. Potts, J. Ji, X. Zhao, R. S. Ruoff, and S. Park, ACS Nano 7, 19 (2013).

${ }^{13}$ R. Lv, G. Chen, Q. Li, A. McCreary, A. Bottelo-Méndez, S. V. Morozov, L. Liang, X. Declerck, N. Perea-López, D. A. Cullen, S. Feng, et al., Proc. Nat. Acad. Sci. 112, 14527 (2015).

${ }^{14}$ D. Y. Yeom, W. Jeon, N. D. K. Tu, S. Y. Yeo, S. S. Lee, B. J. Sung, H. Chang, J. A. Lim, and H. Kim, Sci. Rep. 5, 9817 (2015).

${ }^{15}$ Y. A. Kim, K. Fujisawa, H. Muramatsu, T. Hayashi, M. Endo, T. Fujimori, K. Kaneko, M. Terrones, J. Behrends, A. Eckmann et al., ACS Nano 6, 6293 (2012). ${ }^{16}$ Y. Ota, J. Electrochem. Soc. 124, 1795 (1977).

${ }^{17}$ G. Lippert, J. Dabrowski, Y. Yamamoto, F. Herziger, J. Maultzsch, J. Baringhaus, C. Tegenkamp, M. C. Lemme, and W. Mehr, Phys. Status Solidi B 249, 2507 (2012).

${ }^{18}$ M. H. Oliveira, Jr., T. Schumann, R. Gargallo-Caballero, F. From, T. Seyller, M. Ramsteiner, A. Trampert, L. Geelhaar, J. M. J. Lopes, and H. Riechert, Carbon 56, 339 (2013).
${ }^{19}$ A. Summerfield, A. Davies, T. S. Cheng, V. V. Korolkov, Y. J. Cho, C. J. Mellor, C. T. Foxon, A. N. Khlobystov, K. Watanabe, T. Taniguchi et al., Sci. Rep. 6, 22440 (2016).

${ }^{20}$ J. M. Wofford, F. Speck, T. Seyller, J. M. J. Lopes, and H. Riechert, J. Appl. Phys. 120, 045309 (2016).

${ }^{21}$ D. N. Hendrickson, J. M. Hollander, and W. L. Jolly, Inorg. Chem. 9, 612 (1970).

${ }^{22}$ M. Belyansky, M. Trenary, and C. Elison, Surf. Sci. Spectra 3, 147 (1994).

${ }^{23}$ T. Schumann, J. M. J. Lopes, J. M. Wofford, M. H. Oliveira, Jr., M. Dubslaff, M. Hanke, U. Jahn, L. Geelhaar, and H. Riechert, J. Crystal Growth 425, 274 (2015).

${ }^{24}$ A. C. Ferrari and D. M. Basko, Nat. Nanotechnol. 8, 235 (2013).

${ }^{25}$ A. Das, S. Pisana, B. Chakraborty, S. Piscanec, S. K. Saha, U. V. Waghmare, K. S. Novoselov, H. R. Krishnamurthy, A. K. Geim, A. C. Ferrari, and A. K. Sood, Nat. Nanotechnol. 3, 210 (2008).

${ }^{26}$ M. B. B. S. Larsen, D. M. A. Mackenzie, J. M. Caridad, P. Bøggild, and T. J. Booth, Microelectron. Eng. 121, 113 (2014).

${ }^{27}$ J. Hwang, M. Kim, D. Campbell, H. A. Alsalman, J. Y. Kwak, S. Shivaraman, A. R. Woll, A. K. Singh, R. G. Hennig, S. Gorantla et al., ACS Nano 7, 385 (2013).

${ }^{28}$ J. D. Albar, A. Summerfield, T. S. Cheng, A. Davies, E. F. Smith, A. N. Khlobystov, C. J. Mellor, T. Taniguchi, K. Watanabe, C. T. Foxon et al., Sci. Rep. 7, 6598 (2017). 